\title{
Exposure assessment of bisphenols among Chinese women during pregnancy: a longitudinal study
}

Jiufeng $\mathrm{Li}^{1, \#}$, Chuangsha $\mathrm{Wu}^{2, \# \text {, Hongzhi Zhao }}{ }^{1}$, Yanqiu Zhou ${ }^{1}$, Guodong Cao ${ }^{1}$, Zhiyi Yang $^{1}$, Yanjun Hong ${ }^{1}$, Shunqing $\mathrm{Xu}^{2}$, Wei Xia ${ }^{2 *}$, Zongwei Cai ${ }^{1 *}$

${ }^{1}$ State Key Laboratory of Environmental and Biological Analysis, Department of Chemistry, Hong Kong Baptist University, Hong Kong SAR, China.

${ }^{2}$ Key Laboratory of Environment and Health, Ministry of Education \& Ministry of Environmental Protection, and State Key Laboratory of Environmental Health, School of Public Health, Tongji Medical College, Huazhong University of Science and Technology, Wuhan, China

* Corresponding author:

Prof. Zongwei Cai

State Key Laboratory of Environmental and Biological Analysis, Department of Chemistry, Hong Kong Baptist University, Hong Kong, P. R. China

Tel.: $+852-34117070$

Fax: 34117348

E-mail: zwcai@hkbu.edu.hk

Dr. Wei Xia,

School of Public Health, Tongji Medical College, Huazhong University of Science and Technology, 13 Hangkong Road, Wuhan 430030, China

E-mail: xiawei@hust.edu.cn

\#Both authors contributed equally to this work.

*The authors declare they have no actual or potential competing financial interests. 
Figure S1. Exposure patterns of three bisphenols (distributions of concentrations and composition profiles) among 941 participants over three trimesters (first trimester: T1, second trimester: $\mathrm{T} 2$, and third trimester: $\mathrm{T} 3$ ).

(A) Box-whisker plots of un-adjusted and SG-adjusted concentrations of BPA, BPF, and BPS. Urinary concentrations are plotted using a logarithmic 10 scales.

(B) Pie charts of SG-adjusted urinary concentrations of BPA, BPF, and BPS

(A)

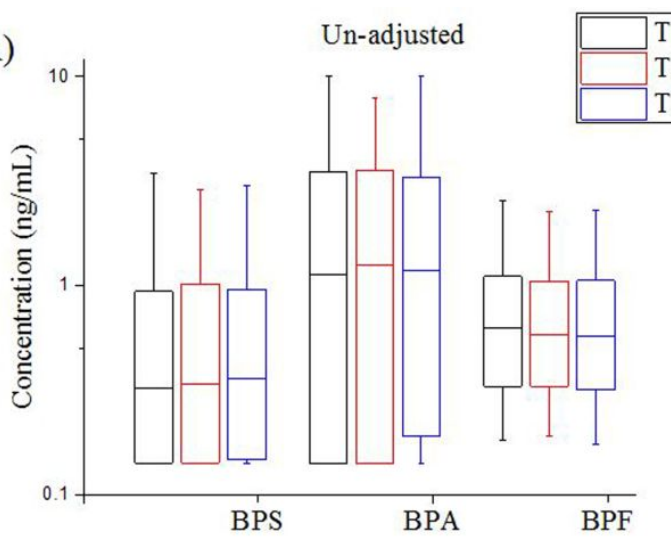

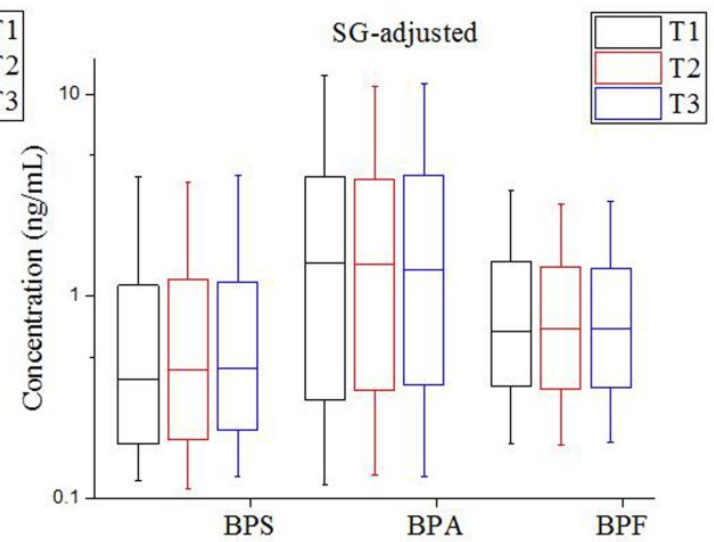

(B)

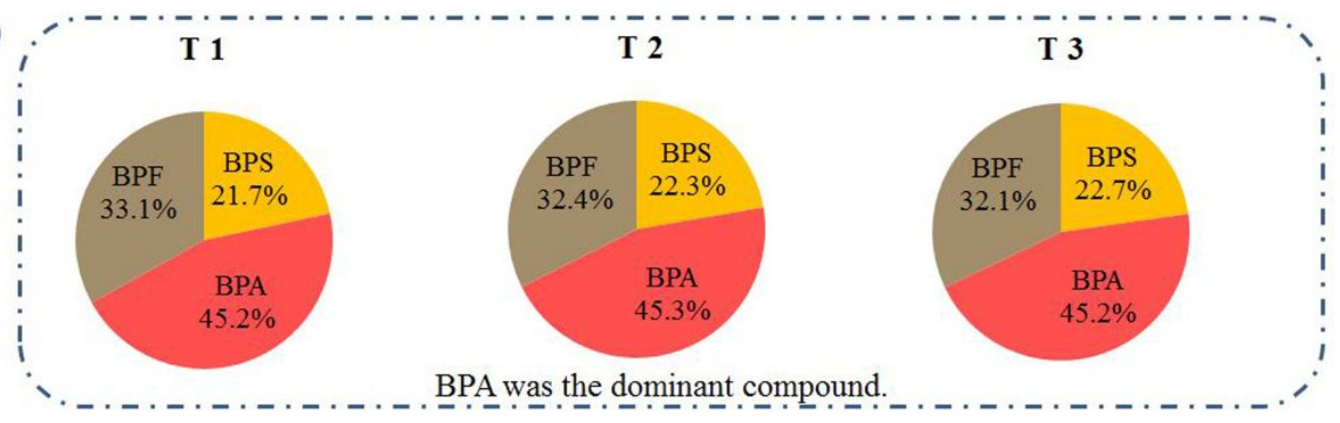


Table S1. Frequency (\% in group) of participants with HI $>1$ and distributions of HI divided by occupation groups

\begin{tabular}{lcccc}
\hline Group & Frequency $(\%)$ & \multicolumn{3}{l}{ HI } \\
\cline { 3 - 5 } & & & & \\
\hline Job & & & & \\
Cashiers & $2(1.71)$ & 0.03 & 0.02 & 0.07 \\
Health care workers & $1(1.96)$ & 0.04 & 0.03 & 0.07 \\
Office workers & $6(1.52)$ & 0.03 & 0.02 & 0.07 \\
Other & $0(0)$ & 0.03 & 0.02 & 0.06 \\
Sales & $3(1.31)$ & 0.03 & 0.02 & 0.06 \\
Teacher & $3(4.11)$ & 0.03 & 0.02 & 0.06 \\
Unemployed & $0(0)$ & 0.03 & 0.02 & 0.05 \\
\hline
\end{tabular}

